\title{
AN UPDATED GSC AS THE ASTROMETRIC REFERENCE FOR MINOR PLANET OBSERVATIONS
}

\author{
SIEGFRIED RÖSER \\ Astronomisches Rechen-Institut \\ Mönchhofstr. 12-14, 69120 Heidelberg, Germany
}

\begin{abstract}
Minor planet observers generally use the Guide Star Catalog (GSC) as the astrometric reference for the reductions of their CCD frames. Two problems are related with GSC. First, it contains no proper motions, and second, it shows severe systematic errors near the plate edges.

I report on the status of a new reduction of GSC and present a first version of STARNET, a catalogue of 4.3 million positions and proper motions using GSC as second, and a new reduction of the Astrographic Catalogue (AC) as first epoch. In STARNET the average rms-accuracy of the proper motions is 5 milliarcsec/year, that of the present-day positions 0.3 ".
\end{abstract}

\section{New GSC reduction}

A comparison between GSC 1.0 (Lasker et al. 1990) and PPM shows large differences with an rms up to 0.7 " especially on the southern hemisphere (Röser et al. 1994). Starting from GSC 1.0 a new plate-by-plate reduction onto the system of PPM has been carried out. This was done by applying a numerical filter with a fixed number (25) of stars (Röser et al. 1994). As a result of this procedure the rms residuals between GSC and PPM coordinates are now better than 0.3 ". The reason for this improvement is a removal of systematic errors on scales smaller than the size of the plates. These were present in GSC 1.0, and were caused by the fact that SAOC and CPC were used as reference catalogues. This improvement was not found by Lopez Garcia and Yagudin (1995), because they only corrected the zero-point of each plate with respect to PPM.

This re-reduction still showed the well-known (Taff et al. 1990) distortions at the plate edges, when residual differences between the newly 
reduced GSC and PPM as functions of the plate coordinates averaged over many plates are formed. From GSC plate-overlaps a radial magnitude equation has been detected. This is confirmed by Morrison, Röser et al. (1995) in a comparison with the Lick Northern Proper Motion Catalog (Klemola et al. 1987). The edge-distortions and the magnitude effect will be removed from GSC within the next few months.

\section{STARNET - the determination of proper motions}

GSC is an observational catalogue. It is not a reference catalogue, because it does not contain proper motions. Galactic rotation and solar motion lead to systematic errors, the larger the more one deviates from the epoch of observation. In some regions of the southern hemisphere, after 20 years of epoch difference, these systematic errors amount to 0.2 ". Referencing observations to GSC means referencing to a coordinate system which rotates at a rate of 5 mas/year.

For about a quarter of the GSC objects, the measurements of the Astrographic Catalogue (AC) can be used as first-epoch observations. These published measurements were put into machine-readable form by Nesterov et al. (1990). Identifying AC with GSC has yielded positions and proper motions for 4.3 million stars. This catalogue of reference stars is called STARNET.

STARNET has the following characteristics: an average star density of 100 stars per square degree, a median magnitude of $B=12.0^{m}$ on the southern hemisphere and $V=11.5^{m}$ on the northern hemisphere. The present-day rms-accuracy of the positions in STARNET is 0.3 ", that of the proper motions 5 mas/year. Comparison of STARNET with observations made with the Bordeaux meridian circle (Requième 1995) confirmed these values, but also showed the systematic errors mentioned in the previous section. STARNET is available at Astronomisches Rechen-Institut. A detailed publication on its construction and its properties is in preparation.

\section{References}

Klemola, A.R., Jones, B.F. and Hanson, R.B., 1987, AJ, 94, 501.

Lasker, B.M., Sturch, C.R., McLean, B.J., Russell, J.L., Jenkner, H. and Shara, M.M., 1990, AJ, 99, 2019.

Lopez Garcia, A. and Yagudin, L.I., 1995, (this volume).

Morrison, J.E., Röser, S., Lasker, B.M., Smart, R.L. and Taff, L.G., 1995, submitted to AJ.

Nesterov V.V., Kislyuk V.S., Potter Kh. I., 1990, In IAU Symposium 141, J.H. Lieske and V.K. Abalakin eds., Kluwer Academic Publishers, Dordrecht, 482.

Röser S., Bastian U., Kuzmin A.N., 1994, in IAU Coll. 148, in press.

Requième, $Y ., 1995$, private communication.

Taff L.G., Lattanzi M.G., Bucciarelli B. et al., 1990, ApJ, 353, L45. 